\title{
Surveying the Best Volatility Measurements to
}

\section{Forecast Stock Market}

\author{
Mohammed Alhagyan ${ }^{*}$, Masnita Misiran² and Zurni Omar ${ }^{3}$ \\ ${ }^{1,2,3}$ Department of Mathematics and Statistics, School of Quantitative Sciences \\ Universiti Utara Malaysia Kedah, Malaysia \\ ${ }^{1}$ Department of Mathematics, Community College in Al-Aflaj \\ Sattam University KSA \\ *Corresponding author
}

Copyright (C) 2017 Mohammed Alhagyan et al. This article is distributed under the Creative Commons Attribution License, which permits unrestricted use, distribution, and reproduction in any medium, provided the original work is properly cited.

\begin{abstract}
This paper investigates methods to forecast future adjusted price of S\&P 500 by using geometric Brownian motion $(\mathrm{GBM})$ and geometric fractional Brownian motion (GFBM) for better investment decision. Four types of formulas are used to find the appropriate volatility measurement that may provide forecast value which closely resembling to actual movement of stock price. The evaluation of forecasting methods is computed by the mean absolute percentage error (MAPE). The findings showed high accuracy in all forecasting methods, with all MAPE are less than $10 \%$, with the best forecasting method is GFBM with stochastic volatility which follow fractional Ornstein - Uhlenbeck (FOU) process.
\end{abstract}

Keywords: Geometric Brownian motion, geometric fractional Brownian motion, volatility, fractional Ornstein- Uhlenbeck process, forecasting

\section{Introduction}

Geometric Brownian motion is considered as one of the most important models and widely used in financial mathematics, in particular as the underlying process of a risky market and able to predict future share price in a short period of time [5]. The parameters in the model may be used in Black-Scholes Model for pricing of options. In recent development, such model evolved by considering the existence of memory, in which the needs to incorporate long memory parameter, 
namely the Hurst index $(H)$ is discussed. Such enhancement of model is better known as the geometric fractional Brownian motion (GFBM).

How best to measure the parameter, i.e., the volatility in the aforementioned model is of the interest in this work.

Volatility is of interest as it discusses the variation in some phenomenon over time. In financial economics, volatility is defined as the standard deviation of the random Wiener driven component in a continuous time diffusion model. However, there are several types of volatility depending on how they are extracted. For example, implied volatility normally extracted from option pricing data, realized volatility from high frequency returns in a day, and historical data from daily returns.

In this work we will forecast the stock market by using GBM and GFBM depending on historical volatility. The volatility measurements that will be used in this work are simple volatility $(S), \log$ volatility $(L)$, high-low-closed $(H L)$, and stochastic volatility $(S T O)$. Detail discussion on the volatility measurements is available in Subsection 4.2.

\section{Derivation of Geometric Fractional Brownian Motion}

We begin this Subsection by constructing geometric Brownian motion, adapted from Abidin and Jaffar [1].

Let $S_{i}$ be asset value on the $i$-th day, and $R_{i}$ be the return from $i$-th day to $i+1$ day which is define as

$$
R_{i}=\frac{S_{i+1}-S_{i}}{S_{i}}
$$

The mean of return or drift $(\mu)$ can be defined as

$$
\mu=\frac{1}{n} \sum_{i=1}^{n} R_{i}
$$

where $n$ is the number of returns in the sample. The standard deviation or volatility, $\sigma$ can be written as

$$
\sigma=\sqrt{\frac{1}{(n-1)} \sum_{i=1}^{n}\left(R_{i}-\mu\right)^{2}} .
$$

Wilott (2000) uses standard normal variables, $\phi$ into the asset return model to standardize the normal distribution of asset return, i.e.,

$$
R_{i}=\frac{s_{i+1}-s_{i}}{S_{i}}=\text { mean }+ \text { standard deviation } \times \phi .
$$

Let $\Delta t$ denotes to time step for one day. By assuming $\mu$ to be constant then:

$$
\text { mean }=\mu \Delta t \text {. }
$$

Let $\sigma$ be a parameter in measuring the amount of randomness, so the standard deviation of the asset returns over time step $\Delta t$ can be written as: 


$$
\text { standard deviation }=\sigma \Delta t^{1 / 2} \text {. }
$$

Consequently,

$$
R_{i}=\frac{S_{i+1}-s_{i}}{S_{i}}=\mu \Delta t+\sigma \phi \Delta t^{1 / 2}
$$

Equation (7) can be rewritten as

$$
S_{i+1}-S_{i}=S_{i} \mu \Delta t+S_{i} \sigma \phi \Delta t^{\frac{1}{2}}
$$

The left hand side in the Equation (8) represents the changes in the asset price, while the right hand side represents the random walk model in discrete time step. As highlighted by Wilmott [12], the stock markets are fluctuating continually over every small interval of time which follows the Brownian motion. Fama [4] argued that the behavior of stock market cannot be foreseen, in which should follow random walk process in GBM. By using the notation of Brownian motion, equation (8) can be written as:

$$
d S(t)=S(t) \mu d t+S(t) \sigma d W_{t}
$$

Equation (9) represents asset price model in continuous-time. $d W_{t}$ represents random variable with mean zero and variance $d t$ which follows

$$
E\left[d W_{t}\right]=0 \quad \text { and } \quad E\left[d W_{t}^{2}\right]=d t
$$

The analytic solution of Equation (9) with initial value $S_{0}$ can be illustrated as

$$
S(t)=S_{0} \operatorname{Exp}\left(\mu t-\frac{1}{2} \sigma^{2} t+\sigma W_{t}\right) .
$$

In what followed, Mishura [7] used fractional Brownian motion, $B_{H}(t)$ to replace the classical Brownian motion $W_{t}$ and further incorporate the existence of long memory in financial market. The suggested model is as follows

$$
d S(t)=S(t) \mu d t+S(t) \sigma d B_{H}(t), \quad H \in\left(\frac{1}{2}, 1\right)
$$

\section{Analytic Solution of GFBM}

Since $H \neq \frac{1}{2}, B_{H}(t)$ is not a semimartingale; thus we cannot apply the general theory of stochastic calculus on $B_{H}(t)$, instead Wick calculus is used.

Now, we assume the initial condition $S(0)=S_{0}$. The stochastic differential equation in Equation (11) can be written as:

$$
\frac{d S(t)}{d t}=S(t) \mu+S(t) \sigma \frac{d B_{H}(t)}{d t} .
$$


By using Wick calculus, Equation (12) can be rewritten in $(\mathcal{S})_{H}^{*}$ as:

$$
\frac{d S(t)}{d t}=S(t) \mu+S(t) \sigma \diamond W_{H}(\mathrm{t})
$$

or

$$
\frac{d S(t)}{d t}=\left(\mu+\sigma \diamond W_{H}(\mathrm{t})\right) S(t) .
$$

Integrate both sides of Equation (14) give

$$
S(t)=S_{0} \operatorname{Exp} \diamond\left(\mu t+\sigma \int_{0}^{t} W_{H}(\mathrm{r}) d r\right)
$$

By using $\frac{d}{d t} B_{H}(t)=W_{H}(t)$ in $(\mathcal{S})_{H}^{*}$, Equation (15) can be written as

$$
S(t)=S_{0} \operatorname{Exp} \diamond\left(\mu t+\sigma B_{H}(t)\right)
$$

Now, we introduce Definition 1, Lemma 1, and Definition 2 from [3] to help with the later deduction.

Definition 1: If $Y: \mathbb{R} \rightarrow(\mathcal{S})_{H}^{*}$ is a given function provided that $Y(t) \diamond W_{H}(t)$ is integrable in $(\mathcal{S})_{H}^{*}$, then we can define the fractional Wick-Ito integral of a function $Y$ as:

$$
\int_{\mathbb{R}} Y(t) d B_{H}=\int_{\mathbb{R}} Y(t) \diamond W_{H}(t) d t
$$

Lemma 1: $\int_{0}^{t} B_{H}(s) d B_{H}(s)=\frac{1}{2} B_{H}^{2}(t)-\frac{1}{2} t^{2 H}$

Definition 2: Let $f \in L_{H}^{2}(\mathbb{R})$, then

$$
\operatorname{Exp} \diamond(<w, f>)=\varepsilon(f)=\operatorname{Exp}\left(\int f d B_{H}-\frac{1}{2}\|f\|_{H}^{2}\right)
$$

By using Definition 2 and Lemma 1, we are ready to illustrate $S(t)$ of GFBM as

$$
S(t)=S_{0} \operatorname{Exp}\left(\mu t+\sigma B_{H}(t)-\frac{1}{2} \sigma^{2} t^{2 H}\right)
$$

Equation (20) represents analytic solution of GFBM based on Wick calculus provided that the volatility is constant. It is also used as asset price modeling that is able to predict asset price at a given time. However, such solution is acceptable given the scenario where volatility is constant. To date, there has yet analytic solution in the case of stochastic volatility.

In this paper, we assume volatility a deterministic function $\sigma\left(Y_{t}\right)$ of stochastic process, $Y_{t}$ which obeys fractional Ornstein-Uhlenbeck process. A more extensive 
discussion on the proposed model is available in [2]. The proposed model is as follows:

$$
\begin{aligned}
& d S_{t}=\mu S_{t} d t+\sigma\left(Y_{t}\right) S_{t} d B_{H_{1}}(t) \\
& d Y_{t}=\alpha\left(m-Y_{t}\right) d t+\beta d B_{H_{2}}(t)
\end{aligned}
$$

where $\alpha, \beta$ and $m$ are constant parameters that represent mean reverting of volatility, volatility of volatility, and mean of volatility respectively. $B_{H_{2}}(t)$ is another fractional Brownian motion. We assume that $B_{H_{1}}(t)$ and $B_{H_{2}}(t)$ are independent.

In the next section, we apply the proposed model in addition to three other models to forecast adjusted prices in S\&P 500 and make some comparison among the different models.

\section{Empirical Investigation}

\subsection{Data}

We used data from S\&P 500. The S\&P 500 is selected for its preferred benchmark of stock market in the US, available online at http://finance.yahoo.com. The daily adjusted closed price from 15th of November 2013 to 31st of December 2013 are selected; with total observation of 30 days. The return series is considered to avoid high volatility in the data.

Figures (1) and (2) show the price and return series of the data set, respectively.

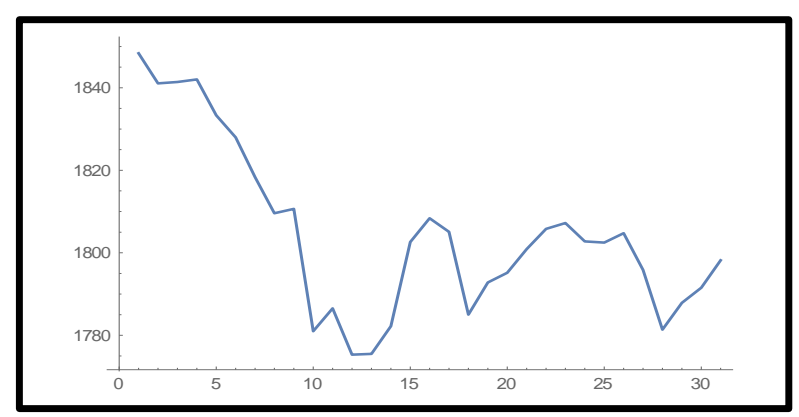

Figure1: Daily adjust price series of S\&P 500 from $15^{\text {th }}$ of November 2013 to 31 of December 2013

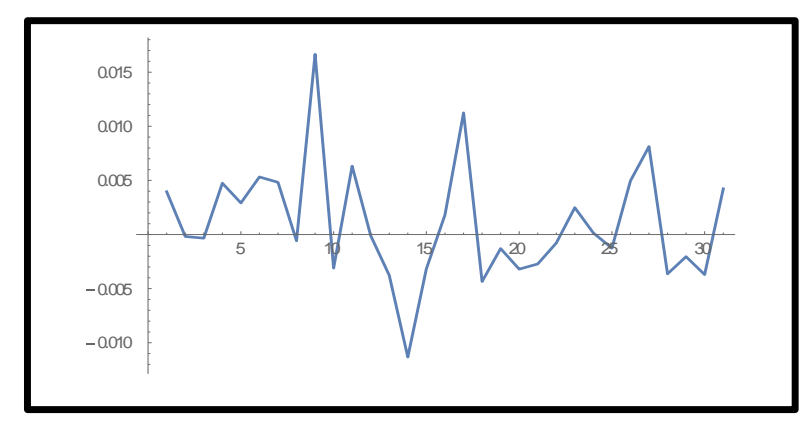

Figure2: Daily returns series of S\&P500 from $15^{\text {th }}$ of November 2013 to 31 of December 2013

In this subsection we forecast the adjusted prices introduced earlier using both GBM and GFBM models. Mathematica 10 software is used to estimate the value of Hurst index $H=0.524$. In this article, we calculate volatility using four different formulas - simple volatility (S), log volatility (L), high-low-closed volatility (HL), and volatility that assumed a deterministic function $\sigma\left(Y_{t}\right)=Y_{t}$ of stochastic process $Y_{t}$ which obeys fractional Ornstein- Uhlenbeck process proposed in this article (STO). 
All S, L and HL depend on constant volatility, whereas STO depends on stochastic volatility. We refer to [12] and [1] for the formulation of S, L and HL:

Simple volatility $(S): \quad \sigma=\sqrt{\frac{1}{(n-1) \Delta t} \sum_{i=1}^{n}\left(R_{i}-\bar{R}\right)^{2}}$

$\log$ volatility $(\boldsymbol{L}): \quad \sigma=\sqrt{\frac{1}{(n-1) \Delta t} \sum_{i=1}^{n}\left(\log \left(S_{i}\right)-\log \left(S_{i-1}\right)\right)^{2}}$

High-Low-Closed volatility (HL):

$\sigma=\sqrt{\frac{1}{(n-1) \Delta t}\left\{\sum_{i=1}^{n} 0.5\left(\log \left(H_{i}\right)-\log \left(L_{i}\right)\right)^{2}-\sum_{i=1}^{n} 0.3\left(\log \left(S_{i}\right)-\log \left(S_{i-1}\right)\right)^{2}\right\}}$

where $n$ is the total of observation, $\Delta t$ is the time step, $R_{i}$ is the return at day $i, S_{i}$ is the price stock at day $i, H_{i}$ is the highest asset price at day $i$ and $L_{i}$ is the lowest asset price at day $i$.

Volatility that obeys FOU (STO): $\quad d Y_{t}=\alpha\left(m-Y_{t}\right) d t+\beta d B_{H_{2}}(t)$

Note that the parameters involved in (21) and (26) are as the follow: $H_{1}=$ $0.5244, H_{2}=0.5289, \mu=0.001024, \beta=2.9 \times 10^{-9}, m=0.000028$ and $\alpha=$ 1.62712 .

Table 1 illustrates different values of volatility according to the selected formula.

Table 1: The values of volatility according to type

\begin{tabular}{ccccc}
\hline $\begin{array}{c}\text { Volatility } \\
\text { type }\end{array}$ & S & L & HL & STO \\
\hline Value & 0.0293275 & 0.0294989 & 0.392966 & 0.029138 \\
\hline
\end{tabular}

Next, we apply the mean absolute percentage error $(M A P E)$ formulation in order to forecast the stock market based on each formulation (Sheldon, 2011) as follows

$$
M A P E=\frac{\sum_{i=1}^{n} \frac{\left|Y_{i}-F_{i}\right|}{Y_{i}}}{n}
$$

Lawrence et.al in [6] determine the scale of judgment of forecast accuracy using MAPE as follows: MAPE $\leq 10 \%$ (highly accurate), 11\% $\leq$ MAPE $\leq 20 \%$ (good accurate), $21 \% \leq$ MAPE $\leq 50 \%$ (reasonable forecast) and MAPE $\geq 51$ (inaccurate).

Table 2 compares the actual prices to the forecast values by using GBM and GFBM model with its four type of volatility and its MAPE. 
Note that in Table $2 \mathrm{~F}_{\text {simple }}$ denoted to forecasting using simple volatility, $\mathrm{F}_{\text {log }}$ denoted to forecasting using log volatility, $\mathrm{F}_{\mathrm{HLC}}$ denoting to Forecasting using high-low-closed volatility and $\mathrm{F}_{\text {Sto. denoting to forecasting using stochastic }}$ volatility.

Table 3 represent the best to the worst of forecasting methods considered in this paper.

Table 2 and Table 3 suggested that most values of MAPE are relatively close, with the values of less than $10 \%$, indicating that both GBM and GFBM models have highly accurate forecasting. However, we can observe that GFBM model is significantly more accurate than GBM model in all cases of different type of volatility. These finding are consistent with [9], [10] and [11] that suggested long memory model to best suited empirical analysis. From the finding, we also learnt that the model proposed in this article produced the most accurate performance whereas GBM model with log volatility performed the worst.

We also noted that all GFBM models performed better in every type of volatility involved. Among different type of volatility in GFBM model, stochastic volatility surpass in its performance as oppose to simple volatility, log volatility and highlow volatility.

\section{Discussion}

In this article, a review of derivation of GFBM is presented by adopting the Wick calculus. A new model of GFBM is introduced, where its volatility is assumed to be a deterministic function $\sigma\left(Y_{t}\right)$ of a stochastic process, $Y_{t}$ which obeys fractional Ornstein-Uhlenbeck process as illustrated in Equation (21) and (22).

We then forecasted prices of S\&P 500 using GBM and GFBM model and investigate its performance. The volatility of the said models follows formulations of simple volatility, log volatility, high-low-closed volatility and volatility of stochastic process. MAPE were computed to evaluate each forecasts.

The findings showed that the proposed method in this article provides the best forecast suggested by its minimum value of $M A P E$. Furthermore, the findings also suggested that GFBM model is significantly more accurate than GBM model, may be due to its long memory property. All forecasting methods also portray high accuracy since all MAPE are less than 10\%. The results are illustrated in Table 2 and Table 3.

Such promising findings motivate more extensive future works on promoting stochastic volatility, GFBM model and long memory in the financial environments. 
Table 2: Forecast Prices and Actual Prices of S\&P 500 with MAPE

\begin{tabular}{|c|c|c|c|c|c|c|c|c|c|}
\hline Date & & & $F_{t}$ & & & & $\boldsymbol{F}$ & & \\
\hline 2014 & $\overline{\text { GBM }}$ & GFBM & $\overline{\text { GBM }}$ & GFBM & $\overline{\text { GBM }}$ & GFBM & GBM & GFBM & Actual \\
\hline 2-Jan & 1846.98 & 1848.29 & 1850.63 & 1848.29 & 1846.86 & 1848.61 & 1846.98 & 1848.28 & 1833.21 \\
\hline 3-Jan & 1852.12 & 1849.07 & 1855.88 & 1849.08 & 1853.78 & 1849.68 & 1852.09 & 1849.06 & 1832.31 \\
\hline 6-Jan & 1844.69 & 1847.61 & 1848.43 & 1847.61 & 1843.79 & 1847.69 & 1844.71 & 1847.61 & 1828.71 \\
\hline 7-Jan & 1849.95 & 1847.68 & 1853.83 & 1847.68 & 1850.86 & 1847.81 & 1849.93 & 1847.67 & 1837.9 \\
\hline 8-Jan & 1844.1 & 1852.02 & 1847.75 & 1852.04 & 1842.99 & 1853.66 & 1844.13 & 1851.99 & 1839 \\
\hline 9-Jan & 1847.25 & 1851.99 & 1850.97 & 1852.02 & 1847.16 & 1853.56 & 1847.25 & 1851.97 & 1840.06 \\
\hline 10-Jan & 1849.76 & 1850.54 & 1853.42 & 1850.56 & 1850.55 & 1851.59 & 1849.74 & 1850.52 & 1841.26 \\
\hline 13-Jan & 1849.75 & 1847.47 & 1853.28 & 1847.47 & 1850.52 & 1847.45 & 1849.74 & 1847.47 & 1821.36 \\
\hline 14-Jan & 1848.01 & 1844.81 & 1851.46 & 1844.79 & 1848.2 & 1843.9 & 1848.01 & 1844.83 & 1840.52 \\
\hline 15-Jan & 1849.87 & 1847.54 & 1853.48 & 1847.54 & 1850.64 & 1847.57 & 1849.86 & 1847.54 & 1847.99 \\
\hline 16-Jan & 1850.29 & 1848.86 & 1854.02 & 1848.86 & 1851.24 & 1849.31 & 1850.27 & 1848.85 & 1844.23 \\
\hline 17-Jan & 1848.09 & 1844.08 & 1851.71 & 1844.06 & 1848.33 & 1842.98 & 1848.08 & 1844.1 & 1841.05 \\
\hline 21-Jan & 1846.08 & 1848.96 & 1849.9 & 1848.97 & 1845.62 & 1849.48 & 1846.09 & 1848.95 & 1844.71 \\
\hline 22-Jan & 1852.46 & 1848.31 & 1856.09 & 1848.31 & 1854.18 & 1848.61 & 1852.43 & 1848.3 & 1842.29 \\
\hline 23-Jan & 1849.55 & 1847.73 & 1853.57 & 1847.73 & 1850.25 & 1847.82 & 1849.54 & 1847.73 & 1826.96 \\
\hline 24-Jan & 1847.81 & 1846.07 & 1851.55 & 1846.06 & 1847.92 & 1845.6 & 1847.81 & 1846.08 & 1791.03 \\
\hline 27-Jan & 1848.64 & 1850.03 & 1852.24 & 1850.04 & 1849.05 & 1850.93 & 1848.63 & 1850.02 & 1783 \\
\hline 28-Jan & 1849.2 & 1851.22 & 1852.87 & 1851.24 & 1849.79 & 1852.51 & 1849.19 & 1851.2 & 1790.15 \\
\hline 29-Jan & 1846.53 & 1848.23 & 1850.11 & 1848.23 & 1846.18 & 1848.51 & 1846.54 & 1848.22 & 1777.17 \\
\hline 30-Jan & 1843.45 & 1850.3 & 1846.98 & 1850.32 & 1842.06 & 1851.25 & 1843.48 & 1850.28 & 1790.88 \\
\hline 31-Jan & 1845.21 & 1845.65 & 1849. & 1845.64 & 1844.49 & 1845.02 & 1845.23 & 1845.66 & 1782.68 \\
\hline 3-Feb & 1847.74 & 1846.49 & 1851.47 & 1846.49 & 1847.81 & 1846.15 & 1847.74 & 1846.5 & 1743.82 \\
\hline 4-Feb & 1851.68 & 1845.19 & 1855.46 & 1845.18 & 1853.09 & 1844.43 & 1851.65 & 1845.21 & 1753.38 \\
\hline 5-Feb & 1849.5 & 1847.37 & 1853.32 & 1847.37 & 1850.16 & 1847.37 & 1849.49 & 1847.38 & 1752.99 \\
\hline 6-Feb & 1852.62 & 1847.61 & 1856.23 & 1847.61 & 1854.36 & 1847.65 & 1852.59 & 1847.61 & 1776.01 \\
\hline 7-Feb & 1849.67 & 1851.77 & 1853.42 & 1851.8 & 1850.39 & 1853.28 & 1849.65 & 1851.75 & 1796.2 \\
\hline 10-Feb & 1848.41 & 1846.85 & 1852.08 & 1846.85 & 1848.75 & 1846.65 & 1848.41 & 1846.86 & 1800.45 \\
\hline 11-Feb & 1852.08 & 1850.62 & 1856.05 & 1850.64 & 1853.65 & 1851.72 & 1852.06 & 1850.6 & 1820.12 \\
\hline 12-Feb & 1846.67 & 1849.32 & 1850.35 & 1849.33 & 1846.39 & 1849.93 & 1846.68 & 1849.31 & 1814.82 \\
\hline 13-Feb & 1853.2 & 1847.55 & 1856.8 & 1847.55 & 1855.13 & 1847.59 & 1853.17 & 1847.55 & 1828.46 \\
\hline 14-Feb & 1844.17 & 1852. & 1847.81 & 1852.03 & 1843. & 1853.54 & 1844.2 & 1851.98 & 1839.03 \\
\hline MAPE & 0.019259 & 0.019206 & 0.021217 & 0.019209 & 0.019457 & 0.019388 & 0.019256 & 0.019204 & \\
\hline
\end{tabular}


Table 3: The order of MAPE and the corresponding methods of forecasting

\begin{tabular}{lllllllll}
\hline & $\boldsymbol{F}_{\text {Sto. }}$ & $\boldsymbol{F}_{\text {simple }}$ & $\boldsymbol{F}_{\text {log }}$ & $\boldsymbol{F}_{\text {Sto. }}$ & $\boldsymbol{F}_{\text {simple }}$ & $\boldsymbol{F}_{\text {HLC }}$ & $\boldsymbol{F}_{\text {HLC }}$ & \multicolumn{1}{c}{$\boldsymbol{F}_{\text {log }}$} \\
\cline { 2 - 9 } $\boldsymbol{*}$ MAPE & $\mathbf{G F B M}$ & $\mathbf{G F B M}$ & $\mathbf{G F B M}$ & $\mathbf{G B M}$ & $\mathbf{G B M}$ & $\mathbf{G F B M}$ & $\mathbf{G B M}$ & $\mathbf{G B M}$ \\
& $1.9204 \%$ & $1.9206 \%$ & $1.9209 \%$ & $1.9256 \%$ & $1.9259 \%$ & $1.9388 \%$ & $1.9457 \%$ & $2.1217 \%$ \\
\hline
\end{tabular}

Acknowledgements. The authors gratefully acknowledge for sponsorship from the Malaysian Ministry of Higher Education, fundamental research grant scheme so code 11992.

\section{References}

[1] S. N. Z Abidin, M. M Jaafar, Surveying the best volatility measurements in stock market forecasting techniques involving small size companies in Bursa Malaysia, 2012 IEEE Symposium on in Humanities, Science and Engineering Research (SHUSER), (2012), 975-979.

https://doi.org/10.1109/shuser.2012.6269014

[2] M. Alhagyan, M. Misiran, Z. Omar, Estimation of Geometric Fractional Brownian Motion Perturbed by Stochastic Volatility Model, Far East Journal of Mathematical Sciences, 99 (2016), no. 2, 221-235.

https://doi.org/10.17654/ms099020221

[3] F. Biagini, Y. Hu, B. Øksendal, T. Zhang, Stochastic Calculus for Fractional Brownian Motion and Applications, Springer Science \& Business Media, 2008. https://doi.org/10.1007/978-1-84628-797-8

[4] E. Fama, The behavior of stock-market prices, Journal of Business, 38 (1965), 34-105.

[5] G. Ladde, L. Wu, Development of modified geometric Brownian motion models by using stock price data and basic statistics, Nonlinear Analysis: Theory, Methods \& Applications, 71 (2009) no. 12, e1203-e1208

https://doi.org/10.1016/j.na.2009.01.151

[6] K.D. Lawrence, R.K. Klimberg, S.M. Lawrence, Fundamentals of Forecasting Using Excel, Industrial Press Inc., 2009.

[7] Y. Mishura, Stochastic Calculus for Fractional Brownian Motion and Related Processes, Vol. 1929, Springer Science \& Business Media, 2008.

https://doi.org/10.1007/978-3-540-75873-0 
[9] S. Painter, Long-Range Dependence in the Subsurface: Empirical Evidence and Simulation Methods, In Invited paper at the American Geophysical Union 1998 Fall Meeting.

[10] I.Z Rejichi, C. Aloui, Hurst exponent behavior and assessment of the MENA stock markets efficiency, Research in International Business and Finance, 26 (2012) no. 3, 353-370. https://doi.org/10.1016/j.ribaf.2012.01.005

[11] W. Willinger, M.S Taqqu, V. Teverovsky, Stock market prices and longrange dependence, Finance and Stochastics, 3 (1999) no. 1, 1-13.

https://doi.org/10.1007/s007800050049

[12] P. Wilmott, Quantitative Finance, John Wiley \& Son, Ltd, Chichester, 2000.

Received: March 29, 2017; Published: April 24, 2017 